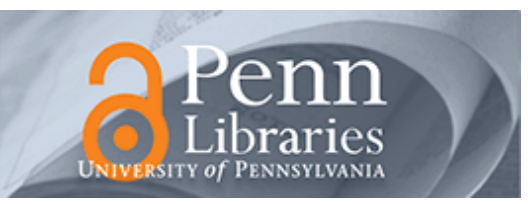

University of Pennsylvania

ScholarlyCommons

\title{
A Distributed Message Passing Computational and I/O Engine for Real-Time Motion Control
}

\author{
M. Buehler \\ Yale University \\ L. L. Whitcomb \\ Yale University \\ F. Levin \\ Yale University
}

Daniel E. Koditschek

University of Pennsylvania, kod@seas.upenn.edu

Follow this and additional works at: https://repository.upenn.edu/ese_papers

Part of the Electrical and Computer Engineering Commons, and the Systems Engineering Commons

\section{Recommended Citation}

M. Buehler, L. L. Whitcomb, F. Levin, and Daniel E. Koditschek, "A Distributed Message Passing Computational and I/O Engine for Real-Time Motion Control", Proceedings of the American Control Conference, 478-483. June 1989.

Copyright 1989 IEEE. Reprinted from Proceedings of the American Control Conference, pages 478-483.

This material is posted here with permission of the IEEE. Such permission of the IEEE does not in any way imply IEEE endorsement of any of the University of Pennsylvania's products or services. Internal or personal use of this material is permitted. However, permission to reprint/republish this material for advertising or promotional purposes or for creating new collective works for resale or redistribution must be obtained from the IEEE by writing to pubs-permissions@ieee.org. By choosing to view this document, you agree to all provisions of the copyright laws protecting it.

NOTE: At the time of publication, author Daniel Koditschek was affiliated with Yale University. Currently, he is a faculty member in the Department of Electrical and Systems Engineering at the University of Pennsylvania.

This paper is posted at ScholarlyCommons. https://repository.upenn.edu/ese_papers/671

For more information, please contact repository@pobox.upenn.edu. 


\title{
A Distributed Message Passing Computational and I/O Engine for Real-Time Motion Control
}

\author{
Abstract \\ This paper illustrates the use of the Yale XP/DCS - a dual board real-time distributed control module \\ based upon the INMOS Transputer family of micoprocessors - for high performance real-time motion \\ control applications. The XP/DCS complements the the Transputer's 1.5 Mflop computational rate and \\ four independent on-chip 20 Mbps DMA communication engines, by providing a bidirectional latched 32 \\ bit bus extension with full handshaking support for easy customization of the $\mathrm{I} / \mathrm{O}$ capabilities of any \\ node. After contrasting this design with commercially available alternatives we describe three particular \\ applications presently underway in the Yale Robotics Laboratory. We conclude by reporting some initial \\ experiments concerning the effect of code distribution and message passing protocols upon sampling \\ rate. \\ For more information: Kod*Lab \\ Disciplines \\ Electrical and Computer Engineering | Engineering | Systems Engineering \\ Comments \\ Copyright 1989 IEEE. Reprinted from Proceedings of the American Control Conference, pages 478-483. \\ This material is posted here with permission of the IEEE. Such permission of the IEEE does not in any way \\ imply IEEE endorsement of any of the University of Pennsylvania's products or services. Internal or \\ personal use of this material is permitted. However, permission to reprint/republish this material for \\ advertising or promotional purposes or for creating new collective works for resale or redistribution must \\ be obtained from the IEEE by writing to pubs-permissions@ieee.org. By choosing to view this document, \\ you agree to all provisions of the copyright laws protecting it. \\ NOTE: At the time of publication, author Daniel Koditschek was affiliated with \\ Yale University. Currently, he is a faculty member in the Department of Electrical \\ and Systems Engineering at the University of Pennsylvania.
}




\title{
A Distributed Message Passing Computational and 1/O Engine for Real-Time Motion Control
}

\author{
M. Bühler, L. L. Whitcomb, F. Levin, D. F. Koditschek ${ }^{1}$ \\ Center for Systems Science \\ Yale University, Department of Electrical Engineering
}

\begin{abstract}
This paper illustrates the use of the Yale XP/DCS - a dualboard real-time distributed control module based upon the IN. MOS Transputer family of microprocessors - for hight perfornance real-time motion control applications. The XP/DCS complements the the 'Transputer's 1.5 Mflop computational rate and four independent on-chip 20 Mbps DMA communcation engines, by providing a bidirectional latched 32 bit bus extension with full handshaking support for easy customization of the $1 / O$ capabilities of any node. After contrasting this design with commercially available altematives we describe three particular applications presently underway in the Yale Robotics I,aboratory. We conclude by reporting some initial experiments concerning the effect of code distribution and message passing protocols upon sampling rate.
\end{abstract}

\section{Introduction}

This paper describes a modular computational engine that has become the workhorse for almost all real-time motion control tasks that we encounter in the Yale Robotics Laboratory. Roughly speaking, these tasks amount to the marshalling of various data - from external sensors; from user specified commands; from motor joint variables - and rapid computation involving their appropriate combinations all in time to produce motor commands that will ensure high performance coordinated movement of many coupled mechanical degrees of freedom.

Our solution to the exigencies of high performance motion control rests largely upon the INMOS "Transputer" fanily of microprocessors. To the powerful built-in computation and communications capabilities of their hardware, INMOS has alded a facile and integrated software/development envitomment extremely well suited to the generation of parallel and concurrent versions of numerically complex algorithms in a message passing environment. Our contribution to the existing technology lies in the design and implementation of a two board set of printed circuits reflecting the $1 / 0$ and memory requirements of the present applications. Our purpose in writing this article is to alert control engineers to the intrinsic capabilities of Transputer networks in general and the Yale "XP/DCS" boardset in particular (Section 2); to provide illustrative examples of the use and performance of our boards in specific motion control applications (Section 3); and to suggest some of the novel theoretical issues that arise when real-time control is eflected by a distributed network of message passing computers (Section 4).

\section{The Yale XP/DCS: Design Require- ments and Alternatives}

Typical servo motors have time constants of between 10 and 100 msec., depending upon the lond. While the theoretically prescribed Nyquist sampling rate is merely double the fastest frequency of the system to be controlled, practical wistom in the control community has long dictated the choice of an order of magnitude higher [5]: we may thus take $1 k \| z z$ as the desired sampling and control update frequency target. The coordination of multiply coupled degrees of freedom exacts a surprisingly high computational cost. For example, an "inverse dynamics" controller for a six tegree of freedom industrial robot matupulator requires roughly $10^{3}$ flops every sampling interval $[6]$. This algorithm, then, would require $1 \mathrm{M} / \mathrm{flop}$ of raw computational capability for real-time implementation. Since the controller must also, sample joint positions and vefocities and deliver torque commands to all six degrees of free. dom, the algorithm assumes that 18 parallel $1 / 0$ operations are performed at the rate of $1 k l l z$ as well. In fact, the computational requirements of robotics applications are unch more stringent if we are interested in autonomous robots and oflyer machines which possess some greater indepentence from luman operators. For example, robot navigation feelback laws for environments cluttered with obstacles [8] involve real-time computation which grows exponentially with the number of degrees of frectom [12]. Intuitively, it is clear that the closer we come to an anthropomorphic motel of the machine, the more overwhelming the real-time computational load.

More relevant, perhaps, to the short term opportunities of the industrial world are recent developments in smart sensor and actuator technology. For example, a new class of "variable reluctance" motors promises relatively large torque/mass ratios at very low speeds suggesting great improvements it direct-drive positioning applications through the elimination of transmissions and linkages. However, satisfaclory performance from these motors requires a great deal of attention to their commutation and current drive strategies [9], necessitating the dedication of a single processor to the production of torque itself in a single motor. Similar computational requirements in smart sensor applications will qutickly convince the forward minded engineer that reat-time motion control applications reguire not merely copious computational capacity but distributed processing and high capracity //O at physically distinct locations.

\footnotetext{
This work is supported in part by hmos Corjoration, GMP Robotics Corporation, PAll Motion 'leclnokgies, Nejtek Corporation, and, in part, by matching funds from the National Science Foundation unter the terms of a Presidential Young Investigator Award held by the last author.
} 
'Two years ago we initiated a search for a powerful, cost effective, and easily expandable solution to the problems of higl performance real-time notion control in the Yale Robotics Laboratory. We sought a system consisting of relatively simple and affordable noles, deployed in a reconfigurable network arrangement with high inter-node bandwidth. Since the theory regarding algoritlum partitioning within a parallel processing environment is still in its infancy, and target control situations are legendary in their variability, we saw system reconfiguration flexibility as the most imporlant goal. Furthermore, since robot sensor and actuator technology is in a state of rapid fux, each node would need to be easily adaptable to specific digital and analog interfacing problems. A most important consideration centered around the software and development environment. We wanted to experiment with motion control algorithms amd emplatically did not want to write real-time operating system kernels or develop our own parallel/concurrent code distribution tools. The system chosen wonld need a stitable hight level language that supports parallel processing, and has capabilities for writing, distributing and debugging code. Most critically, the hardware associated with the development and target system system needed to be affordable.

The options available in the marketplace were numerous when we began our search for solutions two years ago: they are almost bewildering now. Since we feel that our "XP/DCS" system remains attractive today with respect to the required capabilities (and particularly with respect to price/performance criteria) we will present a brief review of commercial alternatives below before describing the nature of our design.

\subsection{Technology Review}

Given clear upper limits on desired processor power and $1 / 0$ capacity it would seem most desirable to use a single centralized CPU. Unfortunately, the open-ended nature of robotics research virtually guarantees that any fixed-capacily computational environment will be quickly exhausted by the ineluctable desire for more degrees of freedom and more complicated algoritlums. 'Thus, a "granular" incrementally costly control architecture seems thavoidable.

\subsubsection{Slared Bus Based Commercial Products}

Commercial motion controllers based upon the current generation of 32 bit microprocessors designed as functional elements on one of the many shared bus arrangements are currently a very popular means to this desirable "finer grain" more flexible and exapandable architecture. As an example consider the offerings from Ironics Inc.,(Ithaca, NY) a VME board manufacturer specializing in multiprocesing applications. Their latest hight-performance board, the IV-900I, is designed around AMD's RISC 29000 microprocessor. With a throughput of 17MIPS at 25MIIz and 6MFLOPS with the 29027 coprocessor, the board has enough processing power to handle the range of tasks in question. The system memory held on a danghter board can lave 2,8 or $16 \mathrm{Mbytes}$ of static-column DRAM. For $1 / 0$ applications they plan a similar daughter board with providing $100 \mathrm{Mbytes} / \mathrm{sec}$ thronghput. Custom $\mathrm{I} / \mathrm{O}$ cards are also possible. The $1 \mathrm{~V}-9001$ base board costs $\$ 7995$. To that must be added memory at $\$ 1995$ for the $2 \mathrm{MB}$ board. The price for the $\mathrm{I} / \mathrm{O}$ danghter board has yet to be announced. The development system consists of a 68020 based Unix workstation coupled to a VME chassis via serial link. Code is witten, compiled and then downloaded to the target boards in the development chassis. Monitors, and debuggers exist to aid in the process. The development system pricing with software starts at $\$ 16,000$.

Other VME boards using the Motorola 68020 and 68030 CISC parts are somewhat less expensive but offer less performance. Ileurikon Corp. (Madison, WI) will provide a $20 \mathrm{MII}$ 68030 VME cpu card with 4Mb DRAM and a $68882 \mathrm{copro}$ cessor for about $\$ 5600$. Witl the Motorola parts, the floating point performance becomes a question for our application. At abont .3 MFLOPS the $68030 / 68882$ won't provide the computational power we desire. Althongh the VME bus has a bandwidth specification of $40 \mathrm{Mbytes} / \mathrm{sec}$, a $680 \times 0$ basel multiprocessor system would probably average no greater than about 3 - 5 Mbytes/sec of avalable inter-processor comminicalions bandwidth. Dificulties arise concerning bus latency and the 'partitioning of code in sucli a system.

Similar considerations apply to the Multibus. As part of the IEEE 1296 specification for the Multibus II, the concept of message passing seeks to accomodate multiple microprocessor systems. A single chip termed the Message Passing Coprocessor was designed by Intel to enable any microprocessor to interface easily to the IEEE 1296 bus. Expected bus bandwidth is improved over that of the VME bus to a respectable $13 \mathrm{Ml}$ ytes/sec in actual applications. Costs for Multibusll boards are still fairly high however. Ilenrikon's HK68/M220 board uses the 32 bit 68020 and has 4MB DRAM, $256 \mathrm{k}$ EPROM and a 68881 coprocessor and lists for $\$ 6295.00$

\subsubsection{Limitations of Shared Bus Architectures}

The high costs per node for these commercial VME and Mulli. bus processor boards represent only one aspect of their deficiency regarding the intended application. For those problems requiring the power of a multiprocesser solution, the major limitations of these systems stem, ironically, from their futtdamental strength, the shared bus architecture, or, as it lias sometimes been described, the "von Neumam! bottleneck." As more processors are added to a system, the bus' ability' to service them diminishes. The point at which the number of processors becomes problematic varies with the application but problems with bus loading and latency are inevilable [11]. Faced with these intrinsic limitations, designers have increas. ingly sought to minimize bus traffic as much as possible. Their solutions take the form of more autonomous "nodes" i.e. pro. cessors having more local resources e.g. dedicated memory and 1/O ports and systems with more eflicient bus handling e.g. the MPC chip on the Multibus II. The enhanced bus handwidth ( $100 \mathrm{MB} / \mathrm{s}$ ) expected with the next generation VME bus, vatiously called Futurebus or Ruggedbus, will provide designers some relief but won't alter the more fundamental constraint limiting performance for multiprocessor systems.

\subsubsection{Summary of Commercial Alternatives}

In summary, whichever of the conventional microprocessors is chosen as the basis of such a customized distributed architecture, all share two big disadvantages. First, they are not designed to be interconnected for parallel processing and tlus, by thenselves, do not afford inter-processor communication. Thus one generally is forced to resort to a bus based approach - the basic structure for almost all parallel real-time control systems built in the past. Unfortunately, the bus commmnication bandwidth decreases at least linearly with the number of nodes and 
1/O units which are attached in a parallel fashion. While it nay be feasible to build such parallel systems with only few nodes, expandability quickly becomes limited (depending on the communication requirements of the specific application). Second, due to the lack of a suitable language, software issues become more and more problematic as the hardware increasingly exploits parallelism.

\subsection{The Yale XP/DCS Controller Node}

In this section we present a description of a system we feel represents a "hest fit" of technology to task: a reconfigurable distributed network of $1 / 0$ customizable boards costing less than $\$ 2000$ each for which a complete off-the-shell commercial tevelopment environment may be purchased for ronghly $\$ 5000$. Moreover, since network links are standardized, our nodes may be trivially interconnected to any of the hundreds of additional existing third party vended boards based upon this technology. We have described the design and performance characteristics of our boards elsewhere in detail $[4,2]$, and will be content with a brief sketch here.

\subsubsection{The INMOS Transputer}

The choice of the INMOS product line represents a strategy which standardizes and places the burden of parallelism inter-processor communications support, software, and development enviromment - aronnd a commercial product, while customizing the computational or $\mathrm{I} / \mathrm{O}$ "identity" of particular nodes by recourse to special purpose hardware.

The Transputer is a 32-bit RISC microprocessor with fast on-chip RAM, interrupt and DMA support, an internal architechre supporting multi-processing, and four high speed DNA serial interprocessor communication links. The latter capability represents the most important feature of this chip relative to its conpetitors. The four links circumvent the constraints of bus based interprocessor communication schemes both with regard to reconfigurability as well as bandwidth. The result is a topology to which nodes are added or deleted simply by physically connecting a four wire serial cable (and a three wire system service connection). Through the parallel processing constructs of the associated programming language, OCCAM, one can equally simply address the software requirements of process concurrency. Whether multitasking on one transputer, or engaged in parallel implementation on a network of transputers, the desired relationships between software processes and hardware processors may be specified with ease and flexibility.

\subsubsection{The XP/DCS Motherboard}

Our motherboard consists of a Transputer chip, 128 Kbytes bytes of zero wait state SRAM, address decoding lardware, and a bus extension connector. We have standardized to the increasingly popular Eurocard form factor, using a board size of $100 \mathrm{~mm} \times 220 \mathrm{~mm}$, the so-called Single Extended Eurocard. The rear edge connector is pin compatible with INMOS' evalnation cards for the It EM system. In particular, the four high speed serial links are made avallable on this rear edge connector. To ensure signal integrity in harsh EMI, two of the links may be routed to fiber optic ports located on the board's front edge. Current hardware choices limit the fiber bandwidth to $5 \mathrm{Nb}$ s, the link speed which will be supported by all transputer family products.
The $l / O$ conncetor located on the lower edge of the hoard, passes the 32 bit Data/Address bus with all interript-, DMA-. and bus-control lines. Thus any add-on board can to be attached that "fits" the transputer memory interface. llowever it. is required that all signals be buffered after the $\mathrm{I} / \mathrm{O}$ connector.

\subsubsection{The XP/DCS I/O board}

Virtually every $\mathrm{I} / \mathrm{O}$ device is now available with tri state inter. face, eliminating the need for a separate latch for each unit, and permitting operation in parallel on a conmon $1 / 0$ bus. Thus, it became soon clear that in order to simultanconsly maximize flexibility as well as ease of use, we lleeded a latched 32 bit bidirectional $1 / 0$ bus which provides for a virtually unlmited number of $1 / O$ devices with minimal chip count. In order to further minimize the custom $1 / 0$ eflort when accommotating a specific $\mathrm{I} / \mathrm{O}$ device, we provide six individually addressable sets of four latched handshaking output lines as well as a total of eight handshaking input lines. In order to prevent arbitrary latched outputs of the handshaking output lines at power up to cause disaster (for example, enabling a robot joint while the torque command is not under control), these ont puls wake up in a high impedance state and can be jumpered to either polarity on the fabricated section of the $1 / O$ boarl. For pro. gramming and debugging convenience, all handshake output lines can be read back.

This implementation provides the user with a maximum of support for custom $1 / 0$ needs: Mlost tristate $1 / 0$ devices should be able to interface to this bus without any adtitional support chips at all. If desired, several devices can be accessed simultaneously: for example, two 16 bit or thee 10 bit bto- $A$ or $A-t o-D$ converters conld be accessed by attaching the different chips to different portions of the $\mathrm{I} / \mathrm{O}$ bus.

We call the mode previously described the asynchmous 1/O mode. Devices can be accessed independent of their speed. A complete $1 / 0$ cycle would take in this default mode would take about $1 \mu \mathrm{s}$, or roughly the time for one flop. Examples of this mode of operation are to be found in Sections 3.1 and 3.3 where quadrature output from shaft encoders is required for motor position control. However, in general the board allows for direct bus interfacing to the transputer in a manner that we call synchronous $\mathrm{I} / \mathrm{O}$ mode. This is possible by removing the bidirectional bus latches. An example of this mode of operation is to be found in Section 3.2 where the $1 / 0$ board carries fast video memory.

\subsubsection{Estimated Cost}

It is perhaps unfair to compare the cost of commercially marketed electronics with "homemade" equipment. Yet we are now using a printed circuit version of the XP/DCS motherboard that has been produced in (small) volume by a local design company for general use in the Yale Robotics Laboratory at a cost of less than $\$ 2000$ for each stuffed and tested board. Surely, given the economies of scale involved in higher volume production, this represents the upper limit of cost that would be incurred by a mass market. A printed circuit version of the XP/DCS 1/O board discussed above is presently in preparation so we have as yet no good estimate of its volume cost (present applications use wire-wrap prototypes). In any event, the daughterboard concept invites customization of $1 / 0$ capability - a very different board has been protolyped for the transmission of video data in the application describe in Section 3.2 - and per board cost will vary greally with the particular design. 


\section{Applications}

The XP/DCS system was designed to be the general workhorse for real-time motion control experiments within the Yale Robotics faboratory. In this section we will briefly review our experiences with three particular robotic devices. References to more elaborate discussions of each apparatus will be given in the text below.

\subsection{A Simple Juggling Robot}

A program of researcli into dexterous manipulation in intermittent dynamical environments $[1,7]$ leads to experimentation with the juggling robot depicted in Figure 1.

The physical apparatus consists of a puck, which slides on an inclined plane and is batted successively by a simple "robot": a bar with billiard cushion rotating in the juggling plane as clepicted in that figure. All intelligent sensor and controller functions are performed by a four node XP/DCS network.

In order to move the bar according to some puck dependent control algorithm, the puck's position and velocity in both directions on the plane must be measured. Presently, this is accomplished by placing an oscillator inside the puck and burying a grid in the juggling plane, thus imitating a big digitizing tablet. On the back of the plane, a simplified XP/DCS system, the Puck Sensor Node, is used as a smart sensor. It measures the voltages inducel in the sensing grid by the puck. The puck position in the plane is computed from the zero and first order moments. 'This information is used to estimate the puck's state: we use a standard linear observer to reduce measurement noise in position and velocity data. Each puck state measurement is communicated asynclironously via fiber optics to the Computation Node. This sampling and communication process is performed at a rate of $1 \mathrm{kllz}$ (when tracking one puck). In the near future we intend to introduce an XI/DCS based real time sterco vision system described in the next section in order to move off the plane into three space. We are fairly confident that the attendant decrease in sampling rate to $60 \mathrm{~Hz}$ will not affect the experimental results significantly.

The Computation Node receives puck state information from the sensor node, reports logging data to the logging node, implements the control algorithm and issues the resulting desired robot states to the Motor Control Node. Varions addilional tasks like detecting the puck motion slatus (up, top, down, impact), predicting puck states (both used for extract. ing logging data) as well as extensive error cleching and housekeeping tasks have to be performed on this note as well. The sampling time can vary between 500 and $1000 \mu \mathrm{s}$. The Motor Control Node is dedicated to commanding a high torque dc servo actuator (built by PMI Motion Technologies) at a rate of $2 \mathrm{kIlz}$.

The experiences with the XP/DCS, the transputer and the development enviromment derived from this application are very encouraging. No single number can capture the ease of use and the little time spent with system overlead. Given the Ts00's intrinsic floating point capability, and the mathematical function library, formulas were programmed (in OCCAM, the native compiler) almost directly from the blackboard with no attempt at code optimization. In spite of substantial calculations, and a great deal of data logging and error landling overhead, very hight sampling rates were achieved. The system operates capably in a high EMI enviromment in consequence of the low cost $5 \mathrm{Mbps}$ fiber optic units from Hewlet Packard built into the Yale XP/DCS boards.

\subsection{A Field Rate Camera System}

The need for teal time stereo vision arises from onr phans to move to a three degree of freedom robot which juggles in three space. The motivation to develop our own vision system as opposed to buying some commercial unit is an outgrowth of that underlying the original XP/DCS design. Most of the computa. tional hardware burden is shouldered by the existing $\mathrm{X} \mathrm{P}^{\mathrm{P}} / \mathrm{DCS}$ node: we need merely provide an effective interface from the video signal to a motherboard throngh the bus extension connector described in Section 2.2. In fact, the prototype working version of the Cyclops system described here was designed and tested by two masters students working in the Vale robotics laboratory on a semester projecl for a compuler archilecture course offerd in the fall of 1988 [13]. Moreover, as is always true of "homebuilt" boards, we end up with a much more powerfu! and flexible system than could be purchased with our modest laboratory equipment grants.

We have successfully tested the system detailed below in a tracking task requiring the position and velocities of two "point masses" moving in three dimensions in structured lighting at field rate (60 IIz). To the best of our knowledge the only commercially available products which achieve this performance $[3,10]$ cost considerably more than the system reported here. 1 We are confident that far more complicated objects may be tracked at near field rates as well, but have not yet developed the necessary software.

Cyclops consists of five main elements as depicted in Figure 2: a camera, a digitizer board, a filter board and one or more video memory boards, each of which connects to a XP/DCS CPUboard. The functions are straight forward the camera provides interlaced fields of video data to the digitizer, which converts the analog signal into an eight bit pixel stream, accompanied with some synchronization signals, The video bus containing the pixel stream is brought to the fitter board. Here any two dimensional filter or convolution with a $6 \times 14$ pixel size window can be applied to the data in real time. Finally the video data is written into one or several video memory "daughter boards" where it can be accessed by an XP/DCS motherboard over the bus connector. All memory boards attach to the video bus in parallel: the maximum possible number is restricted only by drive and noise limitations.

Even though Cyclops satisfies a specific need, it is sufficiently flexible in its operation as to allow its application to most vision tasks. In order to illustrate its generality, we will describe three different distribution schemes for the video fiekds and the implications for update rate and latency. By update rate we mean the rate at which results are computed by any of the processing boards on the video bus whereas latency refers to the total processing time from raw video dat a to end result.

Group Mode: the video fields are londed into all memory boards simulianeously. Depending on the application, each processor then picks a dynamic or static partition of the fill field for processing. A new field is loaded after all processors

\footnotetext{
'Since the vitleo memory boards, unlike the XP/DCS motherboards have not yel been nass produced, it is diffenlt to give a good cost rsti. mate. A complete system incurs rouglıly $\$ 5000$. in electuotics parts - a commercially viable product might be sold for double that amount.
} 
have furished processing - thus latency is equal to update rate.

Scan Mode: for many applications, the video data is not easily separable into partitions without extensive inter-processor communication and decreased performance. In this case one can achieve an increased update rate while kecping the same latency as with only one memory board by sequentially scanning successive fields into different memory boards. Maximally eight memory boards can receive different fields.

Mixcd Morle: this mode combines scan and group mode. Successive fields are scanted into groups of boarls as opposed to into single board as in scan mode. This reduces latency when compared to the scan mode.

\subsection{An Industrial Robot Manipulator}

An alvanced robot controller based on the XP/DCS is under construction for botl testing new robot control algoritlms and investigating issues of distributed real time control. For this task the GMP lobotics Model A-500, a four degree of freedom SCARA type arm, was chosen as the target mechanical unit. $A$ more detailed account of this work may be found in [14].

Like virtually all currently available robot systems, the original A-500 system controller provides an integrated high level user interface which serves admirably in industrial appli. cations, but precludes the low level servo intervention which is needed in the research laboratory. For our experiments it is necessary to be able to directly and independently specify the torque being delivered by each joint of the robot. Since the original control system does not allow this type of interface at any level, it was necessary replace the manufacturer's control system with our own system. For each of the robot's joints, the new interface consists of a delicated XP/DCS node which directly commutates (in sof ware) the currents in the DC brushless motors at the robot joints. The system block diagram for a single joint is shown in Figure 3 .

A primitive protocol has been defined for the interface between the servo transputer and the control network, thus abstracting them as "perfect actuators" which report their state in floating point units of radians, and receive torque conmands in floating point units of Newton-meters. The simple servo 1/O structure gives the designer a clean interface to experiment with high level control algorithm design and implement arbitrary network topologies using standard INMOS compatible nodes. We lave found this approach to offer a powerful and flexible enviromment superior to bus-based multiprocessing enviromments. The speed and simplicity of processor interconnection has proven indispensible in the ease of rapid prototyp. ing and testing of network concepts. The relation of the cont rol network to the low level servo notes is illustrated in Figure 4 which depicts a topology we have found particularly well suited to implementations of the "computed torque" algorithm.

\section{Conclusion}

The XP/DCS has considerably advanced our real-time control capabilities within the Yale Robotics Laboratory both with respect to computational power and simplicity of deployment. We have sketched in Section 2.2 a hardware design based upon the INMOS Transputer yielding a large number of 1.5 Mflop engines, each possessed of zero wait-state direct memory mapped $1 / 0$ capability whose cost (at time of wriling) is less than $\$ 2000$ each. Since typical robotic actuators and intelligent sensors cost at least twice this amount our strategy of assigning (at least) an individual XP/DCS note to each such device can be easily defended against criticisms of profligacy in today's research environment. Given the historically accelerated decreases in electronics cost relative to mechanical lardware we may confidently predict this strategy's commercial cost effectiveness as well within a few years time. We have provicled an incomplete but illustrative review of commercial alternatives to the XP/DCS strategy in Section 2.1 suggesting that our design is less than half the present cost of anything comparable to be found on the market. More critically, as we point ont in that section, given the intended level of granularity in deployment, our Transputer based design scems to offer the only alternative to the contradiction between more processors and higher communications traffic intrinsic to shared bus parallelism.

A uniform, grautar, computationally powerful system of individual nodes with completely reconfigurable interconnection network focnsses research attention upon innovation in algorithm design and physical experimentation rather than cote optimization, operating system hacks, and interface hardware development. The diverse applications presented in Section 3 - a standard industrial manipulator; a frame-rate stereo vision system; a novel juggling robot - suggest just how versatile this notion of a "laboratory workhorse" can be. Since each physical device with its dedicated XP/DCS node las the computational identity of yet another Transputer in the network, the standard INMOS concurrent development environment and network debugging tools may be applied directly. Once a device las been "wedted" to its compulational mate, the integration or re-assignment to another asscmbly or experimental apparatus is no harder than tisconnecting and reconnecting the twisted pair or fiber-optic cables that form the network links.

There do, however, seem to be a number of new theoretical issues concerning the eflicacy of reat-lime controllers whose only mode of communication consists of messages passed around a distributed network (as opposed, for example, to the possibility of "instantaneous" broalcasts or shared menory schemes made possible by the single bus architectures we rejected in Section 2.1). In a recent paper [14] we have added to the familiar ant contrasting notions of updale rate versus latency the slightly more refined distinction between self latemy and cross latency in a distributed real-time message passing controller. Self latency is, rouglly speaking, the average time it takes a given node (or set of nodes) in the control network to adjust the output commands it is producing to the nature of new sensory data received locally. Cross latency, by contrast, is the average time it takes a given node (or sel of notes) to adjust the ontput commands it is producing in response to sensory data received at a distant node (or set of nodes) ant transmitted via a message across the network. We have foumd experimentally that slight variations in code distribution and message passing protocols can have dramatically dilferent ef fect upon the resulting netuork latency matrix. It is to be expected that the the network latency matrix - a much more complicated variation on the familiar theme of controller time constants - will, in turn, have a noticeable impact upon the resulting closed loop behavior of the controlled system. We suspect that the effective design of real-tine controllers built from distributed message passing nodes, attractive thougl such hardware may be, will require a careful analysis of which network latency properties are desirable and how they may be guaranteed by appropriate principles of synthesis. 


\section{Acknowledgements}

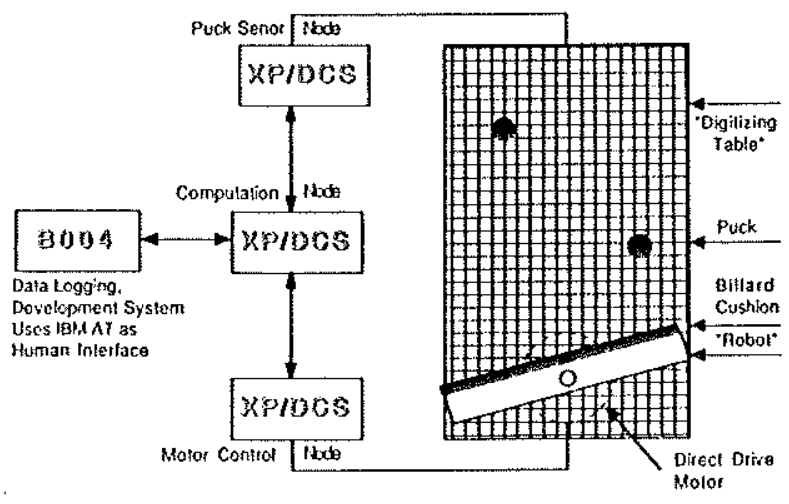

Figure 1: The Yale Juggler

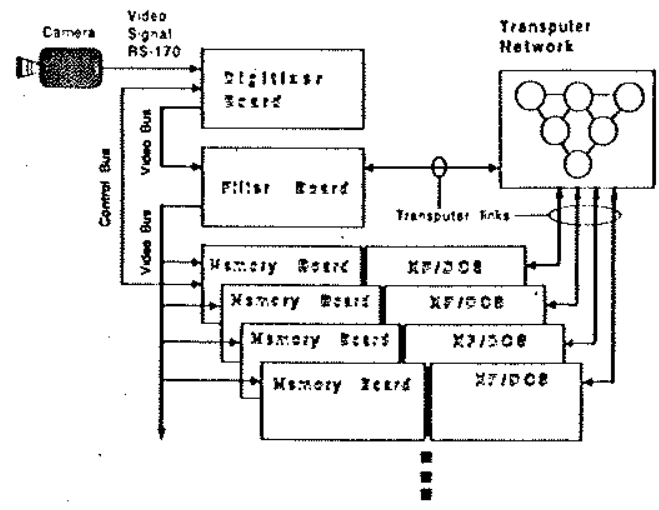

Figure 2: The Cyclops Vision System

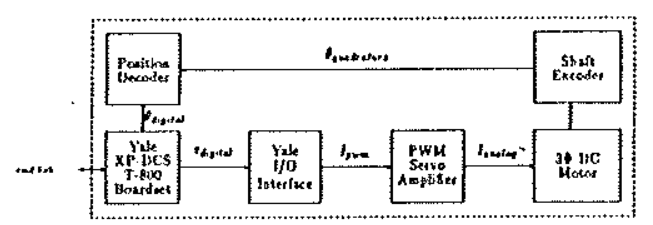

Figure 3: Servo Block Diagram.

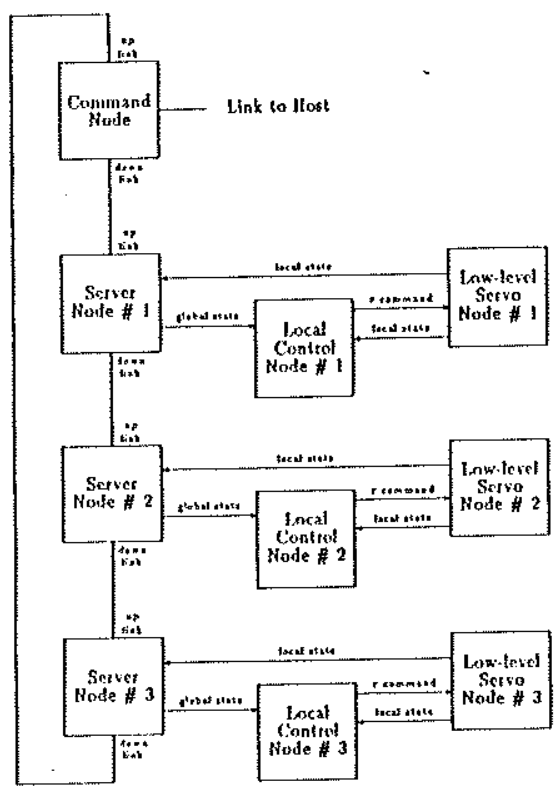

Figure 4: A Sample Control Network Topology.
We would like to thank Professors Peter Kindlmamn and Alfred Ganz for numerous technical and philosophical contributions to the design reported here, as well as their continning generons pedagogical efforts on our behalf.

\section{References}

(1) M. Bühler, D. E. Koditschek, and P.J. Kindlmann. $A$ faurily of robot control strategies for intermittent dymamical environments. In Proc. IEEF Intcrnational Confernor on Robotics and Automation, (to appear), Mrizona, May 1989.

[2] M. Bühler, L. Whilcomb, F. Levin, and D. R. Kodbischek. A new distributed real- time controller for rohotics applica. tions. In Proc. 3/th HEEE Computer Sociely Intermational Conference - COMPCON, pages 63-68, IEEE Computer Sociely Press, San Francisco, CA, Feb 1989.

[3] Datacube. Max Vidco User's Manual. Tednicat leport, Datacube, Inc., \& Dearborn Rd., Peabody, MA 01900, 1986.

[4] F. Levin and M. Bihler and ts. Whitromb and D). F. Koditschek. Transputer computer juggles real-(ine roholics. Electronic Systems Design, 19(2):77-82, Feb 1989.

[5] Gene F. Franklin and J. David Powell. Digital Control of Dynamic Systems. Addison-Wesley, Reading, MA, 1980.

[6] J. M. llollerbach. A recursive formulation of manipulator dynamics and a comparative study of dyuanics formulation and complexity. In Brady et al, editor, Robol Motion, pages 73-87, MIT Press, 1982.

[7] D. E. Koditschek and M. Büller. Analysis of a simplified hopping robot. Int. J. Rob. Research, (to appear), 1989 .

[8] Daniel 1.. Koditscliek and Elon Rimon. Robot navigation functions on manifolds with boundary. Adrances in Applied Mathematics, (to appear).

[9] J. H. Lang, G. C. Verghese, and M. Hic-Spong. Opportunities in estimation and control of electrical motors. In Proc. 25th IEEE Conference on Decision and Control, Athens, Greece, Dec 1986.

[10] Motion Analysis. RTEV Real-Time Camera Mamual. Technical Report, Motion Analysis, Hec., 3650 N. Laughlin Rd., Santa Rosa, CA 95403, 1986.

[11] Per Stenström. Reducing contention in shared-memory multiprocessors. Computer, 21(11):26-37, Nov 1988.

[12] Jacob) 'L. Schwartz and Micla Sharir. On the "Pimo Movers" Problem I. The Case of a Tuo-Dimensional Rigid Polygonal Body Moving I midst Polygonal Barriers. Technical Report 39, N.Y.U. Courant Institute Department of Computer Science, New York, 1981.

[13] C.J. 'Taylor, N. Vlamis, M. Büller, and A. Ganz. The cyclops vision system. In Proc. North Americm Trmsputer Users Group Meeting, Salt Lake City, UT, Apr 1989.

[14] Louis L. Whitcomb, M. Bühler, and D. E. Koditschek. Preliminary experiments real-time distributed motion control. In Proc. North American Transputer Users Group, NY, Oct 1988. 